Mathematical Modelling AND AnAlysis

Volume 18 Number 5, November 2013, 599-611

http://dx.doi.org/10.3846/13926292.2013.865678

(c) Vilnius Gediminas Technical University, 2013
Publisher: Taylor\&Francis and VGTU

http://www.tandfonline.com/TMMA

Print ISSN: 1392-6292

Online ISSN: 1648-3510

\title{
Existence Results for Impulsive Systems with Initial Nonlocal Conditions
}

\section{Octavia Bolojan-Nica ${ }^{a}$, Gennaro Infante ${ }^{b}$ and Paolamaria Pietramala ${ }^{b}$}

${ }^{a}$ Universitatea Babeş-Bolyai

Cluj-Napoca 400084, Romania

${ }^{b}$ Università della Calabria

87036 Arcavacata di Rende, Cosenza, Italy

E-mail: octavia.nica@math.ubbcluj.ro

E-mail(corresp.): gennaro.infante@unical.it

E-mail: pietramala@unical.it

Received March 28, 2013; revised November 7, 2013; published online December 1, 2013

Abstract. We study the existence of solutions for nonlinear first order impulsive systems with nonlocal initial conditions. Our approach relies in the fixed point principles of Schauder and Perov, combined with a vector approach that uses matrices that converge to zero. We prove existence and uniqueness results for these systems. Some examples are presented to illustrate the theory.

Keywords: impulsive differential system, nonlocal initial condition, vector norm, convergent to zero matrix.

AMS Subject Classification: 34A37; 34A12; 34B10; 47H10.

\section{Introduction}

Differential equations with impulses are often used when modelling a variety of phenomena in engineering, physics and life sciences. In the field of population dynamics the impulsive terms model a sudden change in the population size, for example due to stocking or harvesting, for some recent papers in this direction see for example $[1,12,14]$. An introduction to the theory of impulsive differential equations can be found in the books $[3,13,23]$, that contain also a variety of examples.

Here we deal with a system of first order differential equations with impulsive terms subject to nonlocal initial value conditions, namely

$$
\left\{\begin{array}{l}
x^{\prime}(t)=f_{1}(t, x(t), y(t)), \quad y^{\prime}(t)=f_{2}(t, x(t), y(t)), \quad t \in(0,1), t \neq \tau, \\
\left.\Delta x\right|_{t=\tau}=I_{1}(x(\tau)),\left.\quad \Delta y\right|_{t=\tau}=I_{2}(y(\tau)), \quad \tau \in(0,1), \\
x(0)=\alpha_{1}[x], \quad y(0)=\alpha_{2}[y] .
\end{array}\right.
$$


Here $\left.\Delta v\right|_{t=\tau}$ denotes the "jump" of the function $v$ in $t=\tau$, that is

$$
\left.\Delta v\right|_{t=\tau}=v\left(\tau^{+}\right)-v\left(\tau^{-}\right)
$$

where $v\left(\tau^{-}\right), v\left(\tau^{+}\right)$are the left and the right limits of $v$ in $t=\tau$ and $\alpha_{i}$ $(i=1,2)$ are linear functionals given by Stieltjes integrals

$$
\alpha_{i}[v]=\int_{0}^{t_{0}} v(s) d A_{i}(s),
$$

where $t_{0} \in(0, \tau)$ is fixed. The nonlocal conditions (1.2) are fairly general and include, as special cases, $m$-point and integral conditions, when

$$
\alpha_{i}[v]=\sum_{j=1}^{m} \alpha_{i j} v\left(t_{i j}\right) \quad \text { and } \quad \alpha_{i}[v]=\int_{0}^{t_{0}} \alpha_{i}(s) v(s) d s
$$

with $0 \leq t_{i j} \leq t_{0}$. These are widely studied objects, see for example $[2,5,7,8$, $11,15,16,18,19,22,24,25]$, and references therein.

Recently Nica [17] studied the system (1.1) without impulsive terms and $\alpha_{1}, \alpha_{2}$ suitable linear bounded functionals on $C[0,1]$. The methodology in [17] is to rewrite the system as an integral system of the type

$$
\left\{\begin{array}{l}
x(t)=\frac{1}{1-\alpha_{1}[1]} \alpha_{1}\left[g_{1}\right]+g_{1}(x, y)(t), \\
y(t)=\frac{1}{1-\alpha_{2}[1]} \alpha_{2}\left[g_{2}\right]+g_{2}(x, y)(t)
\end{array}\right.
$$

where $1 \neq \alpha_{i}[1]$ and

$$
g_{i}(x, y)(t):=\int_{0}^{t} f_{i}(s, x(s), y(s)) d s, \quad i=1,2,
$$

and to make use of some fixed point theorems combined with matrices that converge to zero and vector-valued norms.

Our idea, similar to the one utilized in $[9,10]$ in the context of secondorder impulsive equations, is to rewrite the system (1.1) as a system of integral equations that can be seen as a perturbation of (1.3), that is

$$
\left\{\begin{array}{l}
x(t)=\frac{1}{1-\alpha_{1}[1]} \alpha_{1}\left[g_{1}\right]+g_{1}(x, y)(t)+G_{1}(x)(t), \\
y(t)=\frac{1}{1-\alpha_{2}[1]} \alpha_{2}\left[g_{2}\right]+g_{2}(x, y)(t)+G_{2}(y)(t),
\end{array}\right.
$$

where the terms $G_{i}$ take into account the impulsive effect.

Here, we benefit also of a careful decomposition similar to the one proposed in $[6]$ and later used in $[17,18]$, namely to rewrite the integral operator associated to the non-impulsive terms as a sum of two operators; one of Fredholm type, whose values depend only on the restrictions to the subinterval $\left[0, t_{0}\right]$, and another one of Volterra type depending on the restrictions to the interval 
$\left[t_{0}, 1\right]$. This allows us to split the growth conditions on the nonlinear terms $f_{1}$ and $f_{2}$ into two parts, one for $t \in\left[0, t_{0}\right]$ and the other one for $\left[t_{0}, 1\right]$. The corresponding conditions in the existence theorems are different in the two intervals, being more relaxed in the last interval. This is the first time that this approach is used in the context of nonlocal impulsive systems.

We present two examples that illustrate the applicability of our results; this is done in the last Section.

\section{Preliminaries}

We now give some notations, definitions and basic results which are used throughout this paper. We make use of the fixed point theorems of Perov and Schauder; in order to apply these theorems we require the notion of convergent to zero matrices (see for example $[20,21]$ ).

Definition 1. A square matrix $M$ with non-negative elements is said to be convergent to zero if

$$
M^{k} \rightarrow 0 \quad \text { as } k \rightarrow \infty .
$$

The next Lemma provides a characterization of matrices converging to zero (see [4, pp. 9, 10], [20,21]).

Lemma 1. Let $M$ be a square matrix of nonnegative numbers. The following statements are equivalent:

(i) $M$ is a matrix that is convergent to zero;

(ii) $I-M$ is nonsingular and $(I-M)^{-1}=I+M+M^{2}+\cdots$ (where $I$ stands for the unit matrix of the same order as $M)$;

(iii) the eigenvalues of $M$ are located inside the unit disc of the complex plane;

(iv) $I-M$ is nonsingular and $(I-M)^{-1}$ has nonnegative elements.

The following lemma is a consequence of the previous characterizations.

Lemma 2. Let $A$ be a matrix that is convergent to zero. Then for each matrix $B$ of the same order whose elements are nonnegative and sufficiently small, matrix $A+B$ is also convergent to zero.

Definition 2. By a vector-valued metric on a set $X$ we mean a mapping $d: X \times X \rightarrow \mathbb{R}_{+}^{n}$ such that

(i) $d(u, v) \geq 0$ for all $u, v \in X$ and if $d(u, v)=0$ then $u=v$;

(ii) $d(u, v)=d(v, u)$ for all $u, v \in X$;

(iii) $d(u, v) \leq d(u, w)+d(w, v)$ for all $u, v, w \in X$;

where, for $x, y \in \mathbb{R}^{n}, x=\left(x_{1}, x_{2}, \ldots, x_{n}\right), y=\left(y_{1}, y_{2}, \ldots, y_{n}\right)$, by $x \leq y$ we mean $x_{i} \leq y_{i}$ for $i=1,2, \ldots, n$. We call the pair $(X, d)$ a generalized metric space. For such spaces convergence and completeness are similar to those in usual metric spaces. 
An operator $T: X \rightarrow X$ is said to be contractive with respect to a vectorvalued metric $d$ on $X$, if there exists a matrix $M$, called Lipschitz matrix, that is convergent to zero such that

$$
d(T(u), T(v)) \leq M d(u, v), \quad \text { for every } u, v \in X .
$$

The following theorem, can be found for example in [20, Theorem 10.1].

Theorem 1 [Perov]. Let $(X, d)$ be a complete generalized metric space and $T: X \rightarrow X$ a contractive operator with Lipschitz matrix $M$. Then $T$ has a unique fixed point $u^{*}$ and for each $u_{0} \in X$ we have

$$
d\left(T^{k}\left(u_{0}\right), u^{*}\right) \leq M^{k}(I-M)^{-1} d\left(u_{0}, T\left(u_{0}\right)\right), \quad \text { for every } k \in \mathbb{N} .
$$

Theorem 2 [Schauder]. Let $X$ be a Banach space, $B \subset X$ a nonempty closed bounded convex set and $T: B \rightarrow B$ a completely continuous operator (i.e., $T$ is continuous and $T(B)$ is relatively compact). Then $T$ has at least one fixed point.

We recall some earlier results of [17] valid for the non-impulsive problem

$$
\left\{\begin{array}{l}
x^{\prime}(t)=f_{1}(t, x(t), y(t)), \quad y^{\prime}(t)=f_{2}(t, x(t), y(t)), \quad t \in(0,1), \\
x(0)=\alpha_{1}[x], \quad y(0)=\alpha_{2}[y]
\end{array}\right.
$$

The approach in [17] is to rewrite the problem (2.1) as an integral system of the type (1.3). The solutions of the system (1.3) are sought as fixed points for the operator

$$
T_{B}(x, y)(t)=\left(\begin{array}{l}
T_{B_{1}}(x, y)(t) \\
T_{B_{2}}(x, y)(t)
\end{array}\right):=\left(\begin{array}{c}
\frac{1}{1-\alpha_{1}[1]} \alpha_{1}\left[g_{1}\right]+g_{1}(x, y)(t) \\
\frac{1}{1-\alpha_{2}[1]} \alpha_{2}\left[g_{2}\right]+g_{2}(x, y)(t)
\end{array}\right),
$$

and the operator $T_{B}$ is decomposed as a sum of two operators, one of Fredholm type and another one of Volterra type, namely

$$
T_{B}=T_{F}+T_{V}
$$

where

$$
T_{F}(x, y)(t)=\left(\begin{array}{c}
T_{F_{1}}(x, y)(t) \\
T_{F_{2}}(x, y)(t)
\end{array}\right), \quad T_{V}(x, y)(t)=\left(\begin{array}{c}
T_{V_{1}}(x, y)(t) \\
T_{V_{2}}(x, y)(t)
\end{array}\right)
$$

with for $i=1,2$

$$
T_{F_{i}}(x, y)(t)= \begin{cases}\frac{1}{1-\alpha_{i}[1]} \alpha_{i}\left[g_{i}\right]+\int_{0}^{t} f_{i}(s, x(s), y(s)) d s, & \text { if } t<t_{0}, \\ \frac{1}{1-\alpha_{i}[1]} \alpha_{i}\left[g_{i}\right]+\int_{0}^{t_{0}} f_{i}(s, x(s), y(s)) d s, & \text { if } t \geq t_{0}\end{cases}
$$

and

$$
T_{V_{i}}(x, y)(t)= \begin{cases}0, & \text { if } t<t_{0} \\ \int_{t_{0}}^{t} f_{i}(s, x(s), y(s)) d s, & \text { if } t \geq t_{0}\end{cases}
$$


A key assumption utilized in the paper [17] is that the matrix

$$
M:=t_{0}\left[\begin{array}{ll}
a_{1}\left(\frac{\left\|\alpha_{1}\right\|}{\mid 1-\alpha_{1}[1]}+1\right) & b_{1}\left(\frac{\left\|\alpha_{1}\right\|}{\mid 1-\alpha_{1}[1]}+1\right) \\
A_{1}\left(\frac{\left\|\alpha_{2}\right\|}{\left|1-\alpha_{2}[1]\right|}+1\right) & B_{1}\left(\frac{\left\|\alpha_{2}\right\|}{\left|1-\alpha_{2}[1]\right|}+1\right)
\end{array}\right]
$$

is converging to zero. This is used in order to apply the theorems of Schauder and Perov for the existence of at least one and for the existence of a unique solution. The matrix $M$ can be written as

$$
M=M_{N}+M_{V}
$$

where

$$
M_{N}:=t_{0}\left[\begin{array}{cc}
a_{1} \frac{\left\|\alpha_{1}\right\|}{\left|1-\alpha_{1}[1]\right|} & b_{1} \frac{\left\|\alpha_{1}\right\|}{\left|1-\alpha_{1}[1]\right|} \\
A_{1} \frac{\left\|\alpha_{2}\right\|}{\left|1-\alpha_{2}[1]\right|} & B_{1} \frac{\left\|\alpha_{2}\right\|}{\left|1-\alpha_{2}[1]\right|}
\end{array}\right] \quad \text { and } \quad M_{V}:=t_{0}\left[\begin{array}{cc}
a_{1} & b_{1} \\
A_{1} & B_{1}
\end{array}\right]
$$

Note that the matrix $M_{N}$ takes into account the nonlocal conditions. The nonnegative coefficients $a_{i}, b_{i}, A_{i}, B_{i}$ are provided by the Lipschitz conditions given by the nonlinearities, namely

$$
\begin{aligned}
& \left|f_{1}(t, x, y)-f_{1}(t, \bar{x}, \bar{y})\right| \leq \begin{cases}a_{1}|x-\bar{x}|+b_{1}|y-\bar{y}|, & \text { if } t \in\left[0, t_{0}\right], \\
a_{2}|x-\bar{x}|+b_{2}|y-\bar{y}|, & \text { if } t \in\left[t_{0}, 1\right],\end{cases} \\
& \left|f_{2}(t, x, y)-f_{2}(t, \bar{x}, \bar{y})\right| \leq \begin{cases}A_{1}|x-\bar{x}|+B_{1}|y-\bar{y}|, & \text { if } t \in\left[0, t_{0}\right], \\
A_{2}|x-\bar{x}|+B_{2}|y-\bar{y}|, & \text { if } t \in\left[t_{0}, 1\right]\end{cases}
\end{aligned}
$$

for all $x, y, \bar{x}, \bar{y} \in \mathbb{R}$.

\section{An existence result}

We now consider the system

$$
\left\{\begin{array}{l}
x^{\prime}(t)=f_{1}(t, x(t), y(t)), \quad y^{\prime}(t)=f_{2}(t, x(t), y(t)), \quad t \in(0,1), t \neq \tau, \\
\left.\Delta x\right|_{t=\tau}=I_{1}(x(\tau)),\left.\quad \Delta y\right|_{t=\tau}=I_{2}(y(\tau)), \quad \tau \in(0,1), \\
x(0)=\alpha_{1}[x], \quad y(0)=\alpha_{2}[y] .
\end{array}\right.
$$

Throughout the paper we assume the following:

(H1) For $i=1,2, f_{i}:[0,1] \times \mathbb{R}^{2} \rightarrow \mathbb{R}$ is such that $f_{i}(., x, y)$ is measurable for each $(x, y) \in \mathbb{R}^{2}$ and $f_{i}(t, .$.$) is continuous for almost all t \in[0,1]$, and for each $r>0$ there exists $\phi_{i, r} \in L^{1}(0,1)$ such that

$$
\left|f_{i}(t, u, v)\right| \leq \phi_{i, r}(t) \text { for } u, v \in[-r, r] \text { and a. e. } t \in[0,1] .
$$

(H2) For $i=1,2$, the function $A_{i}$ is of bounded variation on $\left[0, t_{0}\right]$ with

$$
\alpha_{i}[1]=\int_{0}^{t_{0}} 1 d A_{i}(s) \neq 1 .
$$


(H3) For $i=1,2$, the function $I_{i}: \mathbb{R} \rightarrow \mathbb{R}$ is continuous.

We work in the Banach space $P C_{\tau}[0,1] \times P C_{\tau}[0,1]$, where

$$
\begin{aligned}
P C_{\tau}[0,1]:= & \{u:[0,1] \rightarrow \mathbb{R} \mid, u \text { is continuous in } t \in[0,1] \backslash\{\tau\}, \\
& \text { there exist } \left.u\left(\tau^{-}\right)=u(\tau) \text { and }\left|u\left(\tau^{+}\right)\right|<\infty\right\} .
\end{aligned}
$$

The classical Ascoli-Arzelà compactness criterion cannot be applied directly to the space $P C_{\tau}[0,1]$, here we make use of the following extension of this criterion, see for example [13].

We recall that a set $S \subset P C_{\tau}[0,1]$ is said to be quasi-equicontinuous if for every $u \in S$ and for every $\varepsilon>0$ there exists $\delta>0$ such that $t_{1}, t_{2} \in[0, \tau]$ (or $\left.t_{1}, t_{2} \in(\tau, 1]\right)$ and $\left|t_{1}-t_{2}\right|<\delta$ implies $\left|u\left(t_{1}\right)-u\left(t_{2}\right)\right|<\varepsilon$.

Lemma 1. A set $S \subseteq P C_{\tau}[0,1]$ is relatively compact in $P C_{\tau}[0,1]$ if and only if $S$ is bounded and quasi-equicontinuous.

We use in $P C_{\tau}[0,1] \times P C_{\tau}[0,1]$ the vector norm

$$
\|(x, y)\|_{P C_{\tau}[0,1] \times P C_{\tau}[0,1]}:=(\|x\|,\|y\|),
$$

where

$$
\|v\|:=\max \left\{|v|_{\left[0, t_{0}\right]},\|v\|_{\left[t_{0}, 1\right]}\right\}
$$

and the notation $|v|_{\left[0, t_{0}\right]}$ stands for the sup-norm on $\left[0, t_{0}\right]$ :

$$
|v|_{\left[0, t_{0}\right]}=\sup _{t \in\left[0, t_{0}\right]}|v(t)|
$$

while $\|v\|_{\left[t_{0}, 1\right]}$ denotes a Bielecki-type norm on $\left[t_{0}, 1\right]$ :

$$
\|v\|_{\left[t_{0}, 1\right]}=\sup _{t \in\left[t_{0}, 1\right]}|v(t)| e^{-\theta\left(t-t_{0}\right)}
$$

for some suitable $\theta>0$.

The norm of the functional $\alpha_{i}: P C_{\tau}[0,1] \rightarrow \mathbb{R}$, is given by

$$
\left\|\alpha_{i}\right\|=\sup _{\|v\|=1}\left|\int_{0}^{t_{0}} v(s) d A_{i}(s)\right|
$$

Our idea is to seek a solution of the problem (3.1) as a fixed point of a perturbation of the operator (2.2), namely

$$
T=T_{F}+T_{V}+G
$$

where

$$
G(x, y)(t)=\left(\begin{array}{l}
G_{1}(x)(t) \\
G_{2}(y)(t)
\end{array}\right) \quad \text { and } \quad G_{i}(v)(t)= \begin{cases}0, & \text { if } t \leq \tau \\
I_{i}(v(\tau)), & \text { if } t>\tau\end{cases}
$$

We now show that the existence of solutions for the problem (3.1) follows from Schauder's fixed point theorem when $f_{1}, f_{2}$ satisfy some growth conditions of the type: there exists nonnegative coefficients $a_{i}, b_{i}, c_{i}, A_{i}, B_{i}, C_{i}$ such that 


$$
\begin{aligned}
& \left|f_{1}(t, x, y)\right| \leq \begin{cases}a_{1}|x|+b_{1}|y|+c_{1}, & \text { if } t \in\left[0, t_{0}\right], \\
a_{2}|x|+b_{2}|y|+c_{2}, & \text { if } t \in\left[t_{0}, 1\right],\end{cases} \\
& \left|f_{2}(t, x, y)\right| \leq \begin{cases}A_{1}|x|+B_{1}|y|+C_{1}, & \text { if } t \in\left[0, t_{0}\right], \\
A_{2}|x|+B_{2}|y|+C_{2}, & \text { if } t \in\left[t_{0}, 1\right]\end{cases}
\end{aligned}
$$

for all $x, y \in \mathbb{R}$.

We also assume that there exist $d_{i}, e_{i} \in[0, \infty)$ such that for every $v \in \mathbb{R}$ we have

$$
\left|I_{i}(v)\right| \leq d_{i}|v|+e_{i}, \quad \text { for } i=1,2 .
$$

In what follows we denote by

$$
M_{I}:=\left[\begin{array}{cc}
d_{1} & 0 \\
0 & d_{2}
\end{array}\right] \quad \text { and } \quad A_{\alpha_{i}}:=\frac{1}{\left|1-\alpha_{i}[1]\right|}\left\|\alpha_{i}\right\|+1, \quad i=1,2 .
$$

The matrix $M_{I}$ is essential for our arguments as it takes care of the impulsive effect. This enables us to bridge the methodology employed in [17] to the context of impulsive problems.

Theorem 3. If the conditions (3.3), (3.4), (3.5) are satisfied and the matrix

$$
M_{0}:=M+M_{I}
$$

converges to zero, then the problem (3.1) has at least one solution.

Proof. In order to apply the Schauder fixed point theorem, we look for a nonempty, bounded, closed and convex subset $B$ of $P C_{\tau}[0,1] \times P C_{\tau}[0,1]$ so that $T(B) \subset B$. Let $x, y$ be any elements of $P C_{\tau}[0,1]$.

For $t \in\left[0, t_{0}\right]$, following the proof of Theorem 3.1 of [17], we obtain that

$$
\begin{aligned}
\left|T_{1}(x, y)\right|_{\left[0, t_{0}\right]} & \leq\left(\frac{\| \alpha_{1} \mid}{\left|1-\alpha_{1}[1]\right|}+1\right)\left(a_{1} t_{0}|x|_{\left[0, t_{0}\right]}+b_{1} t_{0}|y|_{\left[0, t_{0}\right]}\right)+c_{1} t_{0} A_{\alpha_{1}} \\
& =a_{1} t_{0} A_{\alpha_{1}}|x|_{\left[0, t_{0}\right]}+b_{1} t_{0} A_{\alpha_{1}}|y|_{\left[0, t_{0}\right]}+c_{1} t_{0} A_{\alpha_{1}} .
\end{aligned}
$$

For $t \in\left[t_{0}, 1\right]$ and any $\theta>0$, we have

$$
\begin{aligned}
\left|T_{1}(x, y)(t)\right| \leq & a_{1} t_{0} A_{\alpha_{1}}|x|_{\left[0, t_{0}\right]}+b_{1} t_{0} A_{\alpha_{1}}|y|_{\left[0, t_{0}\right]}+c_{1} t_{0} A_{\alpha_{1}} \\
& +d_{1}|x(\tau)|+e_{1}+\int_{t_{0}}^{t}\left(a_{2}|x(s)|+b_{2}|y(s)|+c_{2}\right) d s \\
\leq & a_{1} t_{0} A_{\alpha_{1}}|x|_{\left[0, t_{0}\right]}+b_{1} t_{0} A_{\alpha_{1}}|y|_{\left[0, t_{0}\right]}+c_{1} t_{0} A_{\alpha_{1}}+\left(1-t_{0}\right) c_{2}+e_{1} \\
& +d_{1}|x(\tau)| e^{-\theta\left(t-t_{0}\right)} e^{\theta\left(t-t_{0}\right)}+a_{2} \int_{t_{0}}^{t}|x(s)| e^{-\theta\left(s-t_{0}\right)} e^{\theta\left(s-t_{0}\right)} d s \\
& +b_{2} \int_{t_{0}}^{t}|y(s)| e^{-\theta\left(s-t_{0}\right)} e^{\theta\left(s-t_{0}\right)} d s \\
\leq & a_{1} t_{0} A_{\alpha_{1}}|x|_{\left[0, t_{0}\right]}+b_{1} t_{0} A_{\alpha_{1}}|y|_{\left[0, t_{0}\right]}+c_{0} \\
& +d_{1} e^{\theta\left(t-t_{0}\right)}\|x\|_{\left[t_{0}, 1\right]}+\frac{a_{2}}{\theta} e^{\theta\left(t-t_{0}\right)}\|x\|_{\left[t_{0}, 1\right]}+\frac{b_{2}}{\theta} e^{\theta\left(t-t_{0}\right)}\|y\|_{\left[t_{0}, 1\right]}
\end{aligned}
$$


where $c_{0}:=c_{1} t_{0} A_{\alpha_{1}}+\left(1-t_{0}\right) c_{2}+e_{1}$. Dividing by $e^{\theta\left(t-t_{0}\right)}$ and taking the supremum, it follows that

$$
\begin{aligned}
\left\|T_{1}(x, y)\right\|_{\left[t_{0}, 1\right]} \leq & a_{1} t_{0} A_{\alpha_{1}}|x|_{\left[0, t_{0}\right]}+b_{1} t_{0} A_{\alpha_{1}}|y|_{\left[0, t_{0}\right]} \\
& +\left(\frac{a_{2}}{\theta}+d_{1}\right)\|x\|_{\left[t_{0}, 1\right]}+\frac{b_{2}}{\theta}\|y\|_{\left[t_{0}, 1\right]}+c_{0} .
\end{aligned}
$$

Clearly (3.7), (3.8) give

$$
\left\|T_{1}(x, y)\right\| \leq\left(a_{1} t_{0} A_{\alpha_{1}}+d_{1}+\frac{a_{2}}{\theta}\right)\|x\|+\left(b_{1} t_{0} A_{\alpha_{1}}+\frac{b_{2}}{\theta}\right)\|y\|+c_{0} .
$$

Similarly

$$
\left\|T_{2}(x, y)\right\| \leq\left(A_{1} t_{0} A_{\alpha_{2}}+\frac{A_{2}}{\theta}\right)\|x\|+\left(B_{1} t_{0} A_{\alpha_{2}}+d_{2}+\frac{B_{2}}{\theta}\right)\|y\|+C_{0}
$$

with $C_{0}:=C_{1} t_{0} A_{\alpha_{2}}+\left(1-t_{0}\right) C_{2}+e_{2}$.

Now (3.9), (3.10) can be put together as

$$
\left[\begin{array}{l}
\left\|T_{1}(x, y)\right\| \\
\left\|T_{2}(x, y)\right\|
\end{array}\right] \leq M_{\theta}\left[\begin{array}{l}
\|x\| \\
\|y\|
\end{array}\right]+\left[\begin{array}{l}
c_{0} \\
C_{0}
\end{array}\right]
$$

where the matrix $M_{\theta}$ is given by

$$
M_{\theta}=\left[\begin{array}{cc}
A_{\alpha_{1}} a_{1} t_{0}+d_{1}+\frac{a_{2}}{\theta} & A_{\alpha_{1}} b_{1} t_{0}+\frac{b_{2}}{\theta} \\
A_{\alpha_{2}} A_{1} t_{0}+\frac{A_{2}}{\theta} & A_{\alpha_{2}} B_{1} t_{0}+d_{2}+\frac{B_{2}}{\theta}
\end{array}\right] .
$$

Clearly the matrix $M_{\theta}$ can be represented as $M_{\theta}=M_{0}+M_{1}$, where

$$
M_{1}=\left[\begin{array}{cc}
\frac{a_{2}}{\theta} & \frac{b_{2}}{\theta} \\
\frac{A_{2}}{\theta} & \frac{B_{2}}{\theta}
\end{array}\right]
$$

Since $M_{0}$ is assumed to be convergent to zero, from Lemma 1.2 we have that $M_{\theta}$ also converges to zero for large enough $\theta>0$. Next we look for two positive numbers $R_{1}, R_{2}$, such that if $\|x\| \leq R_{1},\|y\| \leq R_{2}$, then $\left\|T_{1}(x, y)\right\| \leq R_{1}$, $\left\|T_{2}(x, y)\right\| \leq R_{2}$. To this end it is sufficient that

$$
\left\{\begin{array}{l}
\left(a_{1} t_{0} A_{\alpha_{1}}+d_{1}+\frac{a_{2}}{\theta}\right) R_{1}+\left(b_{1} t_{0} A_{\alpha_{1}}+\frac{b_{2}}{\theta}\right) R_{2}+c_{0} \leq R_{1}, \\
\left(A_{1} t_{0} A_{\alpha_{2}}+\frac{A_{2}}{\theta}\right) R_{1}+\left(B_{1} t_{0} A_{\alpha_{2}}+d_{2}+\frac{B_{2}}{\theta}\right) R_{2}+C_{0} \leq R_{2}
\end{array}\right.
$$

or equivalently

$$
M_{\theta}\left[\begin{array}{l}
R_{1} \\
R_{2}
\end{array}\right]+\left[\begin{array}{l}
c_{0} \\
C_{0}
\end{array}\right] \leq\left[\begin{array}{l}
R_{1} \\
R_{2}
\end{array}\right]
$$

and therefore

$$
\left[\begin{array}{l}
R_{1} \\
R_{2}
\end{array}\right] \geq\left(I-M_{\theta}\right)^{-1}\left[\begin{array}{l}
c_{0} \\
C_{0}
\end{array}\right]
$$


Notice that $I-M_{\theta}$ is invertible and its inverse $\left(I-M_{\theta}\right)^{-1}$ has nonnegative elements since $M_{\theta}$ converges to zero. Thus, if

$$
B=\left\{(x, y) \in P C_{\tau}[0,1] \times P C_{\tau}[0,1]:\|x\| \leq R_{1},\|y\| \leq R_{2}\right\},
$$

then $T(B) \subset B$. The fact that $T$ is completely continuous follows by Lemma 1 , combined with the useful decomposition (3.2), in a similar way as in the proof of the Theorem 3.3 of [10].

The result now follows from Schauder's fixed point theorem.

Remark 1. From the proof of the Theorem 3 it follows that there exists $\bar{\theta}$ such that the obtained fixed point $(x, y)$ of the operator $T$ satisfies the relation

$$
\|x\|=\max \left\{|x|_{\left[0, t_{0}\right]},\|x\|_{\left[t_{0}, 1\right]}\right\} \leq R_{1},\|y\|=\max \left\{|y|_{\left[0, t_{0}\right]},\|y\|_{\left[t_{0}, 1\right]}\right\} \leq R_{2} .
$$

This implies that, in the interval $\left[0, t_{0}\right]$, we have that

$$
|x(t)| \leq R_{1}, \quad|y(t)| \leq R_{2}
$$

and in $\left[t_{0}, 1\right]$, we have that

$$
|x(t)| \leq R_{1} e^{\bar{\theta}\left(t-t_{0}\right)}, \quad|y(t)| \leq R_{2} e^{\bar{\theta}\left(t-t_{0}\right)} .
$$

We note that a choice of $\theta>\bar{\theta}$ provides a worse estimate in (3.12).

\section{An existence and uniqueness result}

Here, by means of the fixed point theorem of Perov, we prove an existence and uniqueness result, provided that $f_{1}, f_{2}$ satisfy the Lipschitz conditions

$$
\begin{aligned}
& \left|f_{1}(t, x, y)-f_{1}(t, \bar{x}, \bar{y})\right| \leq \begin{cases}a_{1}|x-\bar{x}|+b_{1}|y-\bar{y}|, & \text { if } t \in\left[0, t_{0}\right], \\
a_{2}|x-\bar{x}|+b_{2}|y-\bar{y}|, & \text { if } t \in\left[t_{0}, 1\right]\end{cases} \\
& \left|f_{2}(t, x, y)-f_{2}(t, \bar{x}, \bar{y})\right| \leq \begin{cases}A_{1}|x-\bar{x}|+B_{1}|y-\bar{y}|, & \text { if } t \in\left[0, t_{0}\right] \\
A_{2}|x-\bar{x}|+B_{2}|y-\bar{y}|, & \text { if } t \in\left[t_{0}, 1\right]\end{cases}
\end{aligned}
$$

and also

$$
\left|I_{i}(v)-I_{i}(\bar{v})\right| \leq d_{i}|v-\bar{v}| \quad \text { for } i=1,2,
$$

for all $x, y, \bar{x}, \bar{y}, v, \bar{v} \in \mathbb{R}$.

Theorem 4. If the conditions (4.1), (4.2), (4.3) and the matrix (3.6) converges to zero, then the problem (3.1) has a unique solution.

Proof. We have to prove that $T$ is contractive, that is

$$
\|T(u)-T(\bar{u})\|_{P C_{\tau}[0,1] \times P C_{\tau}[0,1]} \leq M_{\theta}\|u-\bar{u}\|_{P C_{\tau}[0,1] \times P C_{\tau}[0,1]}
$$

for all $u, \bar{u} \in P C_{\tau}[0,1] \times P C_{\tau}[0,1]$ and some matrix $M_{\theta}$ converging to zero. To this end, let $u=(x, y), \bar{u}=(\bar{x}, \bar{y})$ be any elements of $P C_{\tau}[0,1] \times P C_{\tau}[0,1]$. 
For $t \in\left[0, t_{0}\right]$, following the proof of Theorem 2.1 of [17], we have

$$
\begin{aligned}
& \left|T_{1}(x, y)-T_{1}(\bar{x}, \bar{y})\right|_{\left[0, t_{0}\right]} \\
& \quad \leq\left(\frac{|| \alpha_{1} \|}{\left|1-\alpha_{1}[1]\right|}+1\right)\left(a_{1} t_{0}|x-\bar{x}|_{\left[0, t_{0}\right]}+b_{1} t_{0}|y-\bar{y}|_{\left[0, t_{0}\right]}\right) \\
& \quad=A_{\alpha_{1}} a_{1} t_{0}|x-\bar{x}|_{\left[0, t_{0}\right]}+A_{\alpha_{1}} b_{1} t_{0}|y-\bar{y}|_{\left[0, t_{0}\right]} .
\end{aligned}
$$

For $t \in\left[t_{0}, 1\right]$ and any $\theta>0$, we have

$$
\begin{aligned}
&\left|T_{1}(x, y)(t)-T_{1}(\bar{x}, \bar{y})(t)\right| \leq A_{\alpha_{1}} a_{1} t_{0}|x-\bar{x}|_{\left[0, t_{0}\right]}+A_{\alpha_{1}} b_{1} t_{0}|y-\bar{y}|_{\left[0, t_{0}\right]} \\
& \quad+\left|I_{1}(x)(\tau)-I_{1}(\bar{x})(\tau)\right|+\int_{t_{0}}^{t}\left|f_{1}(s, x(s), y(s))-f_{1}(s, \bar{x}(s), \bar{y}(s))\right| d s \\
& \leq A_{\alpha_{1}} a_{1} t_{0}|x-\bar{x}|_{\left[0, t_{0}\right]}+A_{\alpha_{1}} b_{1} t_{0}|y-\bar{y}|_{\left[0, t_{0}\right]} \\
&+d_{1}|x(\tau)-\bar{x}(\tau)|+\int_{t_{0}}^{t}\left(a_{2}|x(s)-\bar{x}(s)|+b_{2}|y(s)-\bar{y}(s)|\right) d s \\
&= A_{\alpha_{1}} a_{1} t_{0}|x-\bar{x}|_{\left[0, t_{0}\right]}+A_{\alpha_{1}} b_{1} t_{0}|y-\bar{y}|_{\left[0, t_{0}\right]}+d_{1}|x(\tau)-\bar{x}(\tau)| e^{-\theta\left(t-t_{0}\right)} e^{\theta\left(t-t_{0}\right)} \\
&+a_{2} \int_{t_{0}}^{t}|x(s)-\bar{x}(s)| \cdot e^{-\theta\left(s-t_{0}\right)} \cdot e^{\theta\left(s-t_{0}\right)} d s \\
&+b_{2} \int_{t_{0}}^{t}|y(s)-\bar{y}(s)| \cdot e^{-\theta\left(s-t_{0}\right)} \cdot e^{\theta\left(s-t_{0}\right)} d s \\
& \leq A_{\alpha_{1}} a_{1} t_{0}|x-\bar{x}|_{\left[0, t_{0}\right]}+A_{\alpha_{1}} b_{1} t_{0}|y-\bar{y}|_{\left[0, t_{0}\right]} \\
&+d_{1} e^{\theta\left(t-t_{0}\right)}\|x-\bar{x}\|_{\left[t_{0}, 1\right]}+\frac{a_{2}}{\theta} e^{\theta\left(t-t_{0}\right)}\|x-\bar{x}\|_{\left[t_{0}, 1\right]}+\frac{b_{2}}{\theta} e^{\theta\left(t-t_{0}\right)}\|y-\bar{y}\|_{\left[t_{0}, 1\right]}
\end{aligned}
$$

Dividing by $e^{\theta\left(t-t_{0}\right)}$ and taking the supremum when $t \in\left[t_{0}, 1\right]$, we obtain

$$
\begin{aligned}
\left\|T_{1}(x, y)-T_{1}(\bar{x}, \bar{y})\right\|_{\left[t_{0}, 1\right]} \leq & A_{\alpha_{1}} a_{1} t_{0}|x-\bar{x}|_{\left[0, t_{0}\right]}+A_{\alpha_{1}} b_{1} t_{0}|y-\bar{y}|_{\left[0, t_{0}\right]} \\
& +\left(\frac{a_{2}}{\theta}+d_{1}\right)\|x-\bar{x}\|_{\left[t_{0}, 1\right]}+\frac{b_{2}}{\theta}\|y-\bar{y}\|_{\left[t_{0}, 1\right]} .
\end{aligned}
$$

Now (4.4) and (4.5) imply that

$$
\left\|T_{1}(x, y)-T_{1}(\bar{x}, \bar{y})\right\| \leq\left(A_{\alpha_{1}} a_{1} t_{0}+d_{1}+\frac{a_{2}}{\theta}\right)\|x-\bar{x}\|+\left(A_{\alpha_{1}} b_{1} t_{0}+\frac{b_{2}}{\theta}\right)\|y-\bar{y}\| .
$$

Similarly we have

$$
\left\|T_{2}(x, y)-T_{2}(\bar{x}, \bar{y})\right\| \leq\left(A_{\alpha_{2}} A_{1} t_{0}+\frac{A_{2}}{\theta}\right)\|x-\bar{x}\|+\left(A_{\alpha_{2}} B_{1} t_{0}+d_{2}+\frac{B_{2}}{\theta}\right)\|y-\bar{y}\| .
$$

Using the vector norm we can put the inequalities $(4.6),(4.7)$ in the form

$$
\|T(u)-T(\bar{u})\|_{P C_{\tau}[0,1] \times P C_{\tau}[0,1]} \leq M_{\theta}\|u-\bar{u}\|_{P C_{\tau}[0,1] \times P C_{\tau}[0,1]},
$$

where $M_{\theta}$ is given by (3.11) and converges to zero for large enough $\theta>0$.

The result follows now from Perov's fixed point theorem. 


\section{$5 \quad$ Numerical examples}

In what follows, we give some numerical examples to illustrate our theory.

Example 1. Consider the initial value problem

$$
\left\{\begin{array}{l}
x^{\prime}=\frac{1}{4} x \sin \left(\frac{y}{x}\right)+\frac{1}{3} y \sin \left(\frac{x}{y}\right)+h_{1}(t) \equiv f_{1}(t, x, y) \\
y^{\prime}=\frac{1}{3} x \sin \left(\frac{y}{x}\right)+\frac{1}{6} y \sin \left(\frac{x}{y}\right)+h_{2}(t) \equiv f_{2}(t, x, y) \\
\left.\Delta x\right|_{t=\frac{3}{4}}=\frac{1}{3} \sin \left(x\left(\frac{3}{4}\right)\right),\left.\quad \Delta y\right|_{t=\frac{3}{4}}=\frac{1}{4} \cos \left(y\left(\frac{3}{4}\right)\right) \\
x(0)=\frac{1}{2} \int_{0}^{\frac{1}{2}} x(s) d s, \quad y(0)=\frac{1}{2} \int_{0}^{\frac{1}{2}} y(s) d s
\end{array} \quad t \in[0,1],\right.
$$

where $h_{1}, h_{2} \in C[0,1]$. We have that $\alpha_{1}[1]=\alpha_{2}[1]=\left\|\alpha_{1}\right\|=\left\|\alpha_{2}\right\|=\frac{1}{4}$. Since

$$
\left|f_{1}(t, x, y)\right| \leq \frac{1}{4}|x|+\frac{1}{3}|y|+\left|h_{1}(t)\right|, \quad\left|f_{2}(t, x, y)\right| \leq \frac{1}{3}|x|+\frac{1}{6}|y|+\left|h_{2}(t)\right|
$$

and $d_{1}=\frac{1}{3}, d_{2}=\frac{1}{4}$, we obtain

$$
M_{0}=\frac{1}{36}\left(\begin{array}{cc}
18 & 8 \\
8 & 13
\end{array}\right),
$$

which is convergent to zero because its eigenvalues (rounded to the third decimal place) are $\lambda_{1}=0.198<1, \lambda_{2}=0.663<1$. From Theorem 3 , the problem (5.1) has at least one solution.

Example 2. We present a modified version of Example 2.2 in [22] that takes into account systems and impulsive effects. Consider the initial value problem

$$
\left\{\begin{array}{l}
x^{\prime}=\frac{1}{2} y\left[1+e^{-\frac{4}{5}(x-1)}\right]^{-1} \equiv f_{1}(x, y) \\
y^{\prime}=\frac{1}{10} x\left[1+e^{-\frac{2}{5}(y-1)}\right]^{-1} \equiv f_{2}(x, y) \\
\left.\Delta x\right|_{t=\frac{3}{4}}=\frac{1}{3} \cos \left(x\left(\frac{3}{4}\right)\right),\left.\quad \Delta y\right|_{t=\frac{3}{4}}=\frac{1}{5} \sin \left(y\left(\frac{3}{4}\right)\right), \quad t \in[0,1] \\
x(0)=\frac{1}{2} \int_{0}^{\frac{1}{2}} x(s) d s, \quad y(0)=\frac{1}{2} \int_{0}^{\frac{1}{2}} y(s) d s
\end{array}\right.
$$

Here we have that $\alpha_{1}[1]=\alpha_{2}[1]=\left\|\alpha_{1}\right\|=\left\|\alpha_{2}\right\|=\frac{1}{4}$. Furthermore we have

$$
\begin{aligned}
& \sup _{x, y \in \mathbb{R}}\left|\frac{\partial f_{1}(x, y)}{\partial x}\right| \leq \frac{1}{10}=a_{1}, \quad \sup _{x, y \in \mathbb{R}}\left|\frac{\partial f_{1}(x, y)}{\partial y}\right| \leq \frac{1}{2}=b_{1}, \\
& \sup _{x, y \in \mathbb{R}}\left|\frac{\partial f_{2}(x, y)}{\partial x}\right| \leq \frac{1}{10}=A_{1}, \quad \sup _{x, y \in \mathbb{R}}\left|\frac{\partial f_{2}(x, y)}{\partial y}\right| \leq \frac{1}{10}=B_{1}
\end{aligned}
$$

and $d_{1}=\frac{1}{3}, d_{2}=\frac{1}{5}$. Therefore the matrix $M_{0}=\frac{1}{15}\left(\begin{array}{ll}6 & 5 \\ 1 & 4\end{array}\right)$ converges to zero since its eigenvalues are $\lambda_{1}=0.17<1, \lambda_{2}=0.5<1$. From Theorem 4 , the problem (5.2) has a unique solution. 


\section{Acknowledgments}

The authors are indebted to the two anonymous referees for their valuable suggestions. This paper was written during the research stage of the first author at the University of Calabria, supported by the Sectorial Operational Programme for Human Resources Development 2007-2013, co-financed by the European Social Fund under project number POSDRU/107/1.5/ S/76841 and title "Modern Doctoral Studies: Internationalization and Interdisciplinarity". The first author was also supported by a grant of the Romanian National Authority for Scientific Research, CNCS - UEFISCDI, project number PN-IIID-PCE-2011-3-0094.

\section{References}

[1] S. Ahmad and I.M. Stamova. Asymptotic stability of an $N$-dimensional impulsive competitive system. Nonlinear Anal. Real World Appl., 8(2):654-663, 2007. http://dx.doi.org/10.1016/j.nonrwa.2006.02.004.

[2] M. Benchohra, E.P. Gatsori, L. Górniewicz and S.K. Ntouyas. Nondensely defined evolution impulsive differential equations with nonlocal conditions. Fixed Point Theory, 4(2):185-204, 2003.

[3] M. Benchohra, J. Henderson and S. Ntouyas. Impulsive Differential Equations and Inclusions, volume 2 of Contemp. Math. Appl. Hindawi Publishing Corporation, New York, 2006. ISBN 977-5945-50-X. http://dx.doi.org/10.1155/9789775945501.

[4] A. Berman and R.J. Plemmons. Nonnegative Matrices in the Mathematical Sciences, volume 9 of Classics Appl. Math. Society for Industrial and Applied Mathematics (SIAM), Philadelphia, PA, 1994. ISBN 0-89871-321-8. http://dx.doi.org/10.1137/1.9781611971262. Revised reprint of the 1979 original

[5] A. Boucherif. Nonlocal Cauchy problems for first-order multivalued differential equations. Electron. J. Differential Equations, pp. No. 47, 9 pp. (electronic), 2002.

[6] A. Boucherif and R. Precup. On the nonlocal initial value problem for first order differential equations. Fixed Point Theory, 4(2):205-212, 2003.

[7] G. Infante, F.M. Minhós and P. Pietramala. Non-negative solutions of systems of ODEs with coupled boundary conditions. Commun. Nonlinear Sci. Numer. Simul., 17(12):4952-4960, 2012. http://dx.doi.org/10.1016/j.cnsns.2012.05.025.

[8] G. Infante and P. Pietramala. Eigenvalues and non-negative solutions of a system with nonlocal BCs. Nonlinear Stud., 16(2):187-196, 2009.

[9] G. Infante and P. Pietramala. Nonlocal impulsive boundary value problems with solutions that change sign. In Mathematical Models in Engineering, Biology and Medicine, volume 1124 of AIP Conf. Proc., pp. 205-213. Amer. Inst. Phys., Melville, NY, 2009. http://dx.doi.org/http://dx.doi.org/10.1063/1.3142935.

[10] G. Infante, P. Pietramala and M. Zima. Positive solutions for a class of nonlocal impulsive BVPs via fixed point index. Topol. Methods Nonlinear Anal., 36(2):263-284, 2010. 
[11] T. Jankowski. Positive solutions for second order impulsive differential equations involving Stieltjes integral conditions. Nonlinear Anal., 74(11):3775-3785, 2011. http://dx.doi.org/10.1016/j.na.2011.03.022.

[12] J.-J. Jiao, L.-S. Chen, J.J. Nieto and A. Torres. Permanence and global attractivity of stage-structured predator-prey model with continuous harvesting on predator and impulsive stocking on prey. Appl. Math. Mech. (English Ed.), 29(5):653-663, 2008. http://dx.doi.org/10.1007/s10483-008-0509-x.

[13] V. Lakshmikantham, D.D. Baĭnov and P.S. Simeonov. Theory of Impulsive Differential Equations, volume 6 of Series in Modern Applied Mathematics. World Scientific Publishing Co. Inc., Teaneck, NJ, 1989. ISBN 9971-50-970-9.

[14] Z. Luo and J.J. Nieto. New results for the periodic boundary value problem for impulsive integro-differential equations. Nonlinear Anal., 70(6):2248-2260, 2009. http://dx.doi.org/10.1016/j.na.2008.03.004.

[15] R. Ma. A survey on nonlocal boundary value problems. Appl. Math. E-Notes, 7:257-279, 2007.

[16] O. Nica. Existence results for second order three-point boundary value problems. Differ. Equ. Appl, pp. No. 4, 24, 2012.

[17] O. Nica. Initial-value problems for first-order differential systems with general nonlocal conditions. Electron. J. Differential Equations, pp. No. 74, 15, 2012.

[18] O. Nica and R. Precup. On the nonlocal initial value problem for first order differential systems. Stud. Univ. Babeş-Bolyai Math., 56(3):113-125, 2011.

[19] S.K. Ntouyas. Nonlocal initial and boundary value problems: a survey. In Handbook of Differential Equations: Ordinary Differential Equations. Vol. II, pp. 461-557. Elsevier B. V., Amsterdam, 2005.

[20] R. Precup. Methods in Nonlinear Integral Equations. Kluwer Academic Publishers, Dordrecht, 2002. ISBN 1-4020-0844-9.

[21] R. Precup. The role of matrices that are convergent to zero in the study of semilinear operator systems. Math. Comput. Modelling, 49(3-4):703-708, 2009. http://dx.doi.org/10.1016/j.mcm.2008.04.006.

[22] R. Precup and D. Trif. Multiple positive solutions of non-local initial value problems for first order differential systems. Nonlinear Anal., 75(15):5961-5970, 2012. http://dx.doi.org/10.1016/j.na.2012.06.008.

[23] A.M. Samoı̌lenko and N.A. Perestyuk. Impulsive Differential Equations, volume 14 of World Sci. Ser. Nonlinear Sci. Ser. A Monogr. Treatises. World Scientific Publishing Co. Inc., River Edge, NJ, 1995. ISBN 981-02-2416-8. http://dx.doi.org/10.1142/9789812798664.

[24] J.R.L. Webb and G. Infante. Positive solutions of nonlocal boundary value problems: a unified approach. J. London Math. Soc. (2), 74(3):673-693, 2006. http://dx.doi.org/10.1112/S0024610706023179.

[25] J.R.L. Webb and G. Infante. Non-local boundary value problems of arbitrary order. J. Lond. Math. Soc. (2), 79(1):238-258, 2009.

http://dx.doi.org/10.1112/jlms/jdn066. 